\title{
The influence of dry-blending operational parameters on homogeneity of milk formula powder
}

\begin{abstract}
Powdered milk formula is generally manufactured using dry blending/mixing process to homogeneously mix the powdered ingredients, especially in low rate self-sufficient milk producing countries. Mixing can be influenced by several factors: particle size, rotation speed, equipment configuration, mixing volume, mixing angle and the cohesiveness of the material. In this project, the effects of operational parameters and feed preparation in a convective batch ribbon powder mixer are investigated. Whole milk powder, skimmed milk powder, whey protein $45 \%$, maltodextrin, lactose, zinc oxide and ascorbic acid powders were used for the mixing study and the formulation was based on standard infant formula in the market. Four typical infant formulae are prepared. Operational parameters of the mixer are filling level (60, 80 and 100\%) and mixing time (5, 10, 15, 20 and 25 min). Chemical tests were done to ensure that the nutrients are uniformly distributed throughout the mixing batch. The results have shown that the mixing time is affected by the filling level. A better mixing can be obtained at lower filling level (60\% for 10-15 min mixing time). Besides, the results also showedthat powders with smaller particle size (skimmed milk powder, $75.02 \mu \mathrm{m}$ ), cohesive particle and round particle surface produced a better mixing performance. Thus, findings from this work can significantly contribute in comprehending the mixing process, the effects of the raw materials characteristics and furthermore in developing efficient mixing.
\end{abstract}

Keyword: Dry blending; Cow milk powder; Infant formula; Convective mixing 